Final 12/10/99 Submitted to J. Non-Cryst. Solids (International Conference on the Physics of Non-Crystalline Solids)

\title{
Monte Carlo simulations of phosphate polyhedron connectivity in glasses
}

\author{
Todd M. Alam \\ Sandia National Laboratories, Albuquerque, NM 87185-1407 USA
}

RECEIVED

DEC ? ? 1999

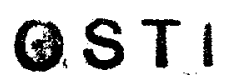

\begin{abstract}
Monte Carlo simulations of phosphate tetrahedron connectivity distributions in alkali and alkaline earth phosphate glasses are reported. By utilizing a discrete bond model, the distribution of nextnearest neighbor connectivities between phosphate polyhedron for random, alternating and clustering bonding scenarios was evaluated as a function of the relative bond energy difference. The simulated distributions are compared to experimentally observed connectivities reported for solid-state two-dimensional exchange and double-quantum NMR experiments of phosphate glasses. These Monte Carlo simulations demonstrate that the polyhedron connectivity is best described by a random distribution in lithium phosphate and calcium phosphate glasses.
\end{abstract}




\section{DISCLAIMER}

This report was prepared as an account of work sponsored by an agency of the United States Government. Neither the United States Government nor any agency thereof, nor any of their employees, make any warranty, express or implied, or assumes any legal liability or responsibility for the accuracy, completeness, or usefulness of any information, apparatus, product, or process disclosed, or represents that its use would not infringe privately owned rights. Reference herein to any specific commercial product, process, or service by trade name, trademark, manufacturer, or otherwise does not necessarily constitute or imply its endorsement, recommendation, or favoring by the United States Government or any agency thereof. The views and opinions of authors expressed herein do not necessarily state or reflect those of the United States Government or any agency thereof. 


\section{DISCLAIMER}

Portions of this document may be illegible in electronic image products. Images are produced from the best available original document. 


\section{Introduction}

Phosphate glasses are technologically important materials for a variety of applications including nuclear waste form encapsulants, glass-to-metal seals, optical devices and biomedical components.[1-5] The continued interest in understanding the relationship between structure and function has resulted in extensive structural investigations of phosphate glass systems. Solid-state nuclear magnetic resonance (NMR) spectroscopy has proven to be a powerful tool for the investigation of both local and medium range order (MRO) in amorphous systems.[6-8] Several NMR active nuclei are available to probe the local structure in phosphate glasses, including ${ }^{31} \mathrm{P}$ magic angle spinning (MAS) NMR investigations of the phosphate backbone, $[8-11]{ }^{27} \mathrm{Al}$ MAS NMR of aluminophosphate glasses,[12] plus ${ }^{23} \mathrm{Na}$ and ${ }^{6,7} \mathrm{Li}$ MAS NMR investigations of the cation environment.[13-15] Information about the extended range connectivity within the phosphate network has recently been obtained using two-dimensional (2D) NMR exchange experiments, by reintroduction of the dipolar coupling during the mixing period or through $2 \mathrm{D}$ double-quantum (2Q) MAS NMR techniques. For some phosphate glasses and ceramics both 2D exchange experiments using the radio frequency dipolar recoupling (RFDR) sequence and 2Q 2D MAS NMR experiments have been reported.[16-27].

A variety of different structural models have been proposed for phosphate glasses, and have been used to explain the experimentally observed connectivities of the phosphate polyhedron determined from 2D NMR techniques. The $Q^{n}$ notation is commonly used to identify and describe the different phosphate tetrahedral species within the glass. For the $\mathrm{Q}^{n}$ notation, $n$ represents the number of bridging oxygens attached to the phosphate (where $n=0,1,2,3$ ), and can range from the fully polymerized $\mathrm{Q}^{3}$ tetrahedral species in $\mathrm{P}_{2} \mathrm{O}_{5}$ to the depolymerized $\mathrm{Q}^{0}$ orthophosphate. Additional superscripts can be included to describe the next-nearest neighbor bonding distribution as detailed below. Three limiting case scenarios or structural models can be presented to describe phosphate polyhedron connectivity; including a random bonding 
distribution of $\mathrm{Q}^{n}$ phosphate species, a structure incorporating alternation of $\mathrm{Q}^{n}$ and $\mathrm{Q}^{n}$ species, or a structure with preferential segregation or clustering of similar $Q^{n}$ phosphate species. All of these limiting scenarios have been forwarded to describe the observed RFDR and 2Q NMR results.

Unfortunately, the majority of NMR investigations to date have evaluated these structural models in a superficial and qualitative manner in my opinion. There has not been a detailed investigation that predicts the network connectivity or next-nearest neighbor distribution that would be observed for these different structural models. Alam and Brow [21] recently derived the distribution of $\mathrm{Q}^{n}$ bonding that would result from a random distribution of bond formation in binary phosphate glasses, but they did not evaluate the distributions for an alternating or clustering model. The ability of different NMR techniques and experiments to distinguish between these limiting case structural models also remains unanswered, and needs to be addressed. In addition, variation of the $\mathrm{Q}^{n}$ polyhedron connectivity distribution for a structure that is described by a combination of these different models also needs to be evaluated.

In this paper the discrete bond model (DBM) proposed by Sprenger et al.[28] was implemented using Monte Carlo (MC) simulations to predict the phosphate tetrahedron connectivity in alkali and earth-alkali phosphate glasses. We performed simulations of the three limiting-case structural models; random, alternating and like species clustering. By varying the relative energy differences between similar $\mathrm{Q}^{n}-\mathrm{O}-\mathrm{Q}^{n}$, and dissimilar $\mathrm{Q}^{n}-\mathrm{O}-\mathrm{Q}^{n}{ }^{n}$ type bonds, we were able to evaluate the changes in the distributions of different connectivity patterns and corresponding NMR observables over a continuous range from the pure alternating structure to the pure clustering structure. 


\section{Computational details}

Monte Carlo simulations were performed by allowing bond formation between 8192 phosphate $\mathrm{Q}^{n}$ species placed on a non-constrained 3D lattice, with no long-range steric effects included. The concentration of $\mathrm{Q}^{n}$ and $\mathrm{Q}^{n^{\prime}}$ species was derived from the glass compositions assuming a simple binary glass system. The $\mathrm{MC}$ simulations were allowed to continue until all bonding connectivity constraints were fulfilled, followed by identification of the distribution of next-nearest connectivities. The probability of bond formation was obtained utilizing a discrete bond model (DBM)[28] to determine the relative bond energies $(\Delta E)$ ( details given the results

section). This bonding probability was defined as $P(\Delta E)=e^{-\beta \Delta E}$, where $\beta=1 / k_{B} T$, with $k_{B}$ being the Boltzman constant and $T$ the temperature. The relative energies reported in the results section are given in terms of $k_{B} T$.

\section{Results}

For binary phosphate glasses the addition of a modifying oxide $\left(\mathrm{R}_{2 / \mathrm{n}}^{+\mathrm{n}} \mathrm{O}\right)$ depolymerizes the structure through the production non-bridging oxygens (NBO) from bridging oxygens within cross-linked polyhedra. This depolymerization can be denoted by[29]

$$
\begin{aligned}
& 2 \mathrm{Q}^{3}+\mathrm{R}_{2 / \mathrm{n}}^{\mathrm{n}+} \mathrm{O} \rightarrow 2 \mathrm{Q}^{2}, \\
& 2 \mathrm{Q}^{2}+\mathrm{R}_{2 / \mathrm{n}}^{\mathrm{n}+} \mathrm{O} \rightarrow 2 \mathrm{Q}^{1}, \\
& 2 \mathrm{Q}^{1}+\mathrm{R}_{2 / \mathrm{n}}^{\mathrm{n}+} \mathrm{O} \rightarrow 2 \mathrm{Q}^{0} .
\end{aligned}
$$


The connectivity of the resulting $Q^{n}$ tetrahedral types is expected to vary based on the relative energies for the different bond types present in the glass structure.[28] For a binary phosphate glasses of composition, $x \mathrm{R}_{2 / n}^{\mathrm{n}+} \mathrm{O} \cdot(1-x) \mathrm{P}_{2} \mathrm{O}_{5}$, only the two glass compositional regions where unique $\mathrm{Q}^{n}-\mathrm{Q}^{n^{\prime}}$ distributions occur are of interest; namely $0 \leq x<0.5$ where only $\mathrm{Q}^{3}$ and $\mathrm{Q}^{2}$ species are present, and $0.5 \leq x<0.67$ where $Q^{2}$ and $Q^{1}$ species are present. At higher modifier mole fractions $(x>0.67)$ only $\mathrm{Q}^{1}$ and $\mathrm{Q}^{0}$ phosphate species are present and do not produce different distributions in connectivity. For these two compositional regions the different limiting-case structural models are defined by

Random distribution

$E_{Q^{3}-Q^{3}}=E_{Q^{2}-Q^{2}}=E_{Q^{3}-Q^{2}}, \Delta E=0 ; \quad 0 \leq x<0.5$,

$E_{Q^{2}-Q^{2}}=E_{Q^{1}-Q^{1}}=E_{Q^{2}-Q^{1}}, \Delta E=0 ; \quad 0.5 \leq x<0.67$.

Alternate distribution

$E_{Q^{3}-Q^{2}} \ll E_{Q^{3}-Q^{3}}=E_{Q^{2}-Q^{2}}, \Delta E=E_{Q^{3}-Q^{3}}-E_{Q^{3}-Q^{2}} ; \quad 0 \leq x<0.5$,

$E_{Q^{2}-Q^{1}} \ll E_{Q^{2}-Q^{2}}=E_{Q^{1}-Q^{1}}, \Delta E=E_{Q^{2}-Q^{2}}-E_{Q^{2}-Q^{1}} ; \quad 0.5 \leq x<0.67$.

Cluster distribution

$E_{Q^{3}-Q^{3}}=E_{Q^{2}-Q^{2}} \ll E_{Q^{3}-Q^{2}}, \Delta E=E_{Q^{3}-Q^{2}}-E_{Q^{2}-Q^{2}} ; \quad 0 \leq x<0.5$,

$E_{Q^{2}-Q^{2}}=E_{Q^{1}-Q^{1}} \ll E_{Q^{2}-Q^{1}}, \Delta E=E_{Q^{2}-Q^{1}}-E_{Q^{2}-Q^{2}} ; \quad 0.5 \leq x<0.67$.

In some phosphate glass systems disproportionation reactions lead to the existence of more that two types of $\mathrm{Q}^{n}$ species being present within the glass for a given composition. For example, in the zinc phosphate glass series, $x \mathrm{ZnO} \bullet(1-x) \mathrm{P}_{2} \mathrm{O}_{5}(0.5 \leq x \leq 0.71)$, both the Raman and ${ }^{31} \mathrm{P}$ MAS NMR results indicate some disproportionation of $Q^{1}$ species to produce $Q^{0}$ and $Q^{2}$ species.[30] The connectivity of phosphate polyhedron in these non-binary phosphate glasses is therefore a more complex issue, and will not be pursued here. 


\subsection{Random distribution}

Analytical solutions for the distribution of next-nearest neighbor phosphate connectivity resulting from a random distribution of bond types can be obtained for simple binary glasses. The distribution of connectivities for a mixture of $\mathrm{Q}^{n}$ and $\mathrm{Q}^{\prime \prime}$ species is most easily described by the fraction of $\mathrm{Q}^{n}$ species having differing number of $\mathrm{Q}^{\prime \prime}$ groups directly bonded to it. Following the nomenclature of Witter et. al,[27] this fraction is defined by $f\left(\mathrm{Q}^{n, i j k}\right)$ where $n$ describes the number of bridging oxygen attached to the phosphate tetrahedron, and the additional superscripts $i, j, k$ denote the types of adjacent bonded $\mathrm{Q}^{n^{\prime}}$ tetrahedra. For example, $f\left(\mathrm{Q}^{3,333}\right)$ represents the fraction of $\mathrm{Q}^{3}$ having three $\mathrm{Q}^{3}$ tetrahedra directly bonded, while $f\left(\mathrm{Q}^{2.23}\right)$ represents the fraction of $\mathrm{Q}^{2}$ species that are bonded to a single $\mathrm{Q}^{3}$ and single $\mathrm{Q}^{2}$ tetrahedron.

For modifier concentrations between $0 \leq x<0.5$ only $\mathrm{Q}^{3}$ and $\mathrm{Q}^{2}$ species are present, so the fraction of $Q^{3}$ tetrahedra having $0,1,2$ or $3 Q^{2}$ species directly bonded in a random bond distribution is defined by a binomial distribution $[21,31]$

$$
\begin{aligned}
& f\left(\mathrm{Q}^{3,333}\right)=\left(1-b_{\mathrm{Q}^{2}}\right)^{3}, \\
& f\left(\mathrm{Q}^{3,233}\right)=3 b_{\mathrm{Q}^{2}}\left(1-b_{\mathrm{Q}^{2}}\right)^{2}, \\
& f\left(\mathrm{Q}^{3,223}\right)=3 b_{\mathrm{Q}^{2}}^{2}\left(1-b_{\mathrm{Q}^{2}}\right), \\
& f\left(\mathrm{Q}^{3,222}\right)=3 b_{\mathrm{Q}^{2}}^{3},
\end{aligned}
$$

where $b_{\mathrm{Q}^{2}}$ is the mole fraction of $\mathrm{Q}^{2}$ bonds available. For modifier concentrations between $0 \leq x$ $\leq 0.5$ the mole fraction of $\mathrm{Q}^{3}$ and $\mathrm{Q}^{2}$ bonds is given by 
$b_{Q^{3}}=\frac{3-6 x}{3-4 x}$,

$b_{Q^{2}}=\frac{2 x}{3-4 x}$.

Using Eqs. (5a), (5b), (5c), (5d) and (6b) the $Q^{n}$ connectivities for the $Q^{3}$ phosphate tetrahedron as a function of modifier concentration can be calculated, and are shown in Fig. 1a. Similarly the distribution around the $\mathrm{Q}^{2}$ tetrahedral species is again given by a binomial distribution

$f\left(\mathrm{Q}^{2,22}\right)=\left(1-b_{\mathrm{Q}^{3}}\right)^{2}$,

$f\left(\mathrm{Q}^{2,23}\right)=2 b_{\mathrm{Q}^{3}}\left(1-b_{\mathrm{Q}^{3}}\right)$,

$f\left(\mathrm{Q}^{2,33}\right)=2 b_{\mathrm{Q}^{3}}^{2}$.

Using Eqs. (6a), (7a), (7b) and (7c) the distribution of the $Q^{2}$ species can be calculated and is shown in Fig. 1b. These distribution curves for the random connectivity will be compared to the distributions simulated for other structural models below.

For modifier concentrations between $0.5 \leq x<0.67$, where the glass is composed of only $\mathrm{Q}^{2}$ and $\mathrm{Q}^{1}$ species, the bonding distribution around the $\mathrm{Q}^{2}$ tetrahedral species is given by

$$
\begin{aligned}
& f\left(\mathrm{Q}^{2,22}\right)=\left(1-b_{\mathrm{Q}^{1}}\right)^{2}, \\
& f\left(\mathrm{Q}^{2,12}\right)=b_{\mathrm{Q}^{1}}\left(1-b_{\mathrm{Q}^{1}}\right), \\
& f\left(\mathrm{Q}^{2,11}\right)=b_{\mathrm{Q}^{\prime}}^{2},
\end{aligned}
$$

where the normalized mole fractions of the $\mathrm{Q}^{2}$ and $\mathrm{Q}^{1}$ bonds is given by[32] 
$b_{Q^{2}}=\frac{4-6 x}{3-4 x}$,

$b_{Q^{1}}=\frac{2 x-1}{3-4 x}$.

Using Eqs. (8a), (8b), (8c) and (9b) the distribution of $Q^{2}$ connectivities can be calculated and is shown in Fig. 2a. The distribution around the $Q^{1}$ species is easily. defined by

$f\left(\mathrm{Q}^{1,2}\right)=b_{\mathrm{Q}^{2}}=1-f\left(\mathrm{Q}^{1,1}\right)$.

Using Eqs. (9a) and (10) the variation of the $Q^{1}$ distributions as a function of modifier concentration are shown in Fig. 2b.

\subsection{Alternate connectivity}

The distribution of connectivities assuming that $\mathrm{Q}^{\prime}-\mathrm{Q}^{\prime}(i \neq j)$ bond formation is energetically preferred over $Q^{i}-Q^{i}$ formation (Eqn 3 ) leads to glass structures in which the identity of the $Q^{n}$ species alternates within the network. Alternating $Q^{n}$ structures have been proposed for zinc phosphate glasses.[33] The MC simulation of the $\mathrm{Q}^{n}$ connectivity distributions as a function of differences in relative energies $(\Delta E)$ are shown for the modifier concentrations rangeș, $0 \leq x<$ 0.5 (Figs. 3,4) and $0.5 \leq x<0.67$ (Fig. 5). For the largest $\Delta E$ the resulting structures show alternation of $\mathrm{Q}^{n}$ species, and clearly dominate the distribution (Fig. 3a, 4a, 5a and 5c). For example, below the metaphosphate concentration $(0.0 \leq x<0.5)$, the $f\left(Q^{2,33}\right)$ connectivity fraction (Fig. 4a) is dominant for almost the entire range and only diminishes when the concentration of the $\mathrm{Q}^{3}$ species becomes smaller than $\sim 33 \%$. Similarly, the $f\left(\mathrm{Q}^{3,222}\right.$ ) fraction (Fig. 3a) becomes 
dominant after fulfilling the initial coordination constraints of the $\mathrm{Q}^{3}$ polyhedra. The alternation of $Q^{n}$ species is obtained in lieu of coordination environments that are not fully alternating. For example, the $f\left(\mathrm{Q}^{3,233}\right)$ and $f\left(\mathrm{Q}^{3,223}\right)$ connectivities are not observed in Fig. 3a, nor is the $f\left(\mathrm{Q}^{2,23}\right)$ coordination environment observed in Fig. 4a. As expected, when the energy differences between bond types diminishes ( $\Delta E \rightarrow 0$ ) the observed distributions approach those predicted for a random distribution. Note the similarity between Fig. 3d and 1a, Fig. 4d and 1b, Fig. 5b and 2a, and Fig. $5 \mathrm{~d}$ and $2 \mathrm{~b}$. The MC simulations for the alternating distribution model also reveal that the phosphate connectivities respond differently as a function of $\Delta E$, and therefore provide an additional way of estimating the degree of pure alternating connectivity versus a mixed alternating-random connectivity distribution. For example, as $\Delta E$ 's are reduced to $\sim 1$ through 5 $k_{B} T$, for $0 \leq x<0.5$, the concentration of the $f\left(\mathrm{Q}^{3,233}\right)$ and $f\left(\mathrm{Q}^{3,223}\right)$ connectivities begin to increase (Fig. $3 \mathrm{~b}$ and $3 \mathrm{c}$ ), signaling the breakdown of the pure alternating structure. The same trend is also observed for the concentration of the $f\left(Q^{2.23}\right.$ ) connectivity (Fig. $4 b$ and $4 c$ ). For $0.5 \leq x<0.67$ this breakdown of the alternating connectivity is observed at much smaller $\Delta E$ 's, occurring between $\sim 1$ and $0.5 k_{B} T$ (Fig. 5). The MC simulations for the alternating structural model described above will be compared to the experimentally observed distributions from RFDR and 2Q NMR experiments in the discussion section.

\subsection{Cluster connectivity}

Monte Carlo simulations assuming that $Q^{j}-Q^{i}$ bond formation is energetically preferred over dissimilar $\mathrm{Q}^{i}-\mathrm{Q}^{j}(i \neq j)$ bond formation (Eqn 4$)$ leads to network structures containing clustering of similar $\mathrm{Q}^{n}$ species. The $\mathrm{MC}$ simulations of the resulting connectivity distributions as a function of relative energy differences $(\Delta E)$ are shown for the modifier concentrations ranges, $0.0 \leq x<$ 0.5 (Fig. 6) and $0.5 \leq x<0.67$ (Fig. 7). The impact of the cluster model on the distributions is 
larger than the effect observed for the alternating structural model, and requires much smaller $\Delta E$ 's for deviations from the pure clustering distribution to be observed. For example, when $\Delta E$ is $\geq 2.5 k_{B} T$ (not shown), the $f\left(\mathrm{Q}^{3,333}\right.$ ) and $f\left(\mathrm{Q}^{2,22}\right)$ species dominate with approximately unity for the entire concentration range, $0 \leq x<0.5$, whereas the $f\left(\mathrm{Q}^{2,22}\right)$ and $\left.f \mathrm{Q}^{1.1}\right)$ are the dominant species observed for $0.5 \leq x<0.67$. For $\Delta E<1 k_{B} T$ the distribution becomes quite complex as seen in Fig. 6 and 7. In general, cluster type connectivities are preferred with a suppression of those species that contain alternating connectivity. As noted above, the connectivity distributions approach those distributions predicted for a random model as $\Delta E$ approaches zero (Compare Fig $6 \mathrm{c}$ and $1 \mathrm{a}$, Fig. $6 \mathrm{f}$ and $1 \mathrm{~b}$, Fig. 7c and $2 \mathrm{a}$, Fig. $7 \mathrm{f}$ and $2 \mathrm{~b}$ ). The $\Delta E$ 's obtained from these simulations do not correspond to any experimentally measured $\Delta E$, but do provide a metric for comparison of the uniqueness and mixing of the various structural models. The results of these MC simulations for the clustering structural model will be compared to the experimentally observed distributions from RFDR and 2Q NMR experiments in the discussion.

\section{Discussion}

\subsection{RFDR 2D exchange experiments}

There have been several RFDR 2D exchange investigations reported for phosphate glasses.[16,17,21,22] The majority of these NMR investigations discuss connectivities qualitatively due to concerns of differential exchange rates as well as interference from unexchanged magnetization along the diagonal. Alam and Brow [21] recently reported a more quantitative analysis of 2D RFDR exchange experiments for a series of lithium ultraphosphate glasses. Unfortunately the individual $f\left(\mathrm{Q}^{n i j k}\right)$ connectivities can not be directly measured from $2 \mathrm{D}$ 
exchange spectra. Instead these experiments provide estimates of the relative concentration of $Q^{n}$ $-Q^{n \prime}$ linkages through integration of the exchange cross-peaks at short mixing times.[21] The relative fraction of $\mathrm{Q}^{n}-\mathrm{Q}^{n^{\prime}}$ linkages present in the glass structure can be related to the linear combination of individual $f\left(\mathrm{Q}^{n, i j k}\right)$ values. For $0 \leq x<0.5$, the relative fraction of bridging $\mathrm{P}-\mathrm{O}_{\mathrm{B}}$ bonds in a $Q^{3}$ polyhedron that are attached to $Q^{2}$ species is defined by

$$
P_{\mathrm{Q}^{3}, \mathrm{Q}^{2}}=1-P_{\mathrm{Q}^{3}, \mathrm{Q}^{3}}=\frac{\left[\mathrm{P}^{\mathrm{Q}^{3}}-\mathrm{O}-\mathrm{P}^{\mathrm{Q}^{2}}\right]}{\left[\mathrm{P}-\mathrm{O}_{\mathrm{B}}\right]}=\frac{1}{3} f\left(\mathrm{Q}^{3,233}\right)+\frac{2}{3} f\left(\mathrm{Q}^{3,223}\right)+f\left(\mathrm{Q}^{3,222}\right),
$$

while the fraction of $\mathrm{P}-\mathrm{O}$ bonds in the $\mathrm{Q}^{2}$ polyhedron that are attached to $\mathrm{Q}^{3}$ species is defined by

$$
P_{\mathrm{Q}^{2}, \mathrm{Q}^{3}}=1-P_{\mathrm{Q}^{2}, \mathrm{Q}^{2}}=\frac{\left[\mathrm{P}^{\mathrm{Q}^{2}}-\mathrm{O}-\mathrm{P}^{\mathrm{Q}^{3}}\right]}{\left[\mathrm{P}-\mathrm{O}_{\mathrm{B}}\right]}=\frac{1}{2} f\left(\mathrm{Q}^{2.23}\right)+f\left(\mathrm{Q}^{2,33}\right) .
$$

Similar arguments for the polyphosphate region, $0.5 \leq x<0.67$, lead to the relative populations of $\mathrm{P}-\mathrm{O}$ bonds in the $\mathrm{Q}^{2}$ species that are bonded to $\mathrm{Q}^{1}$ species:

$$
P_{\mathrm{Q}^{2}, \mathrm{Q}^{\mathrm{l}}}=1-P_{\mathrm{Q}^{2}, \mathrm{Q}^{2}}=\frac{\left[\mathrm{P}^{\mathrm{Q}^{2}}-\mathrm{O}-\mathrm{P}^{\mathrm{Q}^{1}}\right]}{\left[\mathrm{P}-\mathrm{O}_{\mathrm{B}}\right]}=\frac{1}{2} f\left(\mathrm{Q}^{2,12}\right)+f\left(\mathrm{Q}^{2,11}\right) .
$$

Because the $\mathrm{Q}^{1}$ species has only a single bonding oxygen, the relative $P_{\mathrm{Q}^{1}, \mathrm{Q}^{2}}$ distribution is simply given by $b_{\mathrm{Q}^{2}}$ (Eqn. 9a). By using Eqs. 5-13 the predicted variation of linkages as a function of modifier concentration can be determined, and are shown in Fig. 8 along with the experimental distributions reported for the lithium ultraphosphate glasses series.[21] For these comparisons the limiting case scenarios were investigated by utilizing large $\triangle E$ during the MC simulations of the 
alternate $\left(\Delta E=10 k_{B} T\right)$ and cluster models $\left(\Delta E=5 k_{B} T\right)$. Fig. 8 shows that only the random distribution describes the experimentally observed intensities of the cross-peaks. The alternating structural model predicts that the $Q^{3} \rightarrow Q^{3}$ (solid line, Fig. 8a) intensity decreases more rapidly than observed experimentally, while $\mathrm{Q}^{3} \rightarrow \mathrm{Q}^{2}$ intensity (dotted line, Fig. 8a) increases more rapidly than the experimental data. For the pure cluster model the $Q^{3} \rightarrow Q^{3}$ (solid line, Fig. 8a) and $Q^{3} \rightarrow Q^{2}$ intensities (dotted line, Fig. 8a) show little variation with modifier concentration, and are near one and zero, respectively. For the $Q^{2} \rightarrow Q^{3}$ and $Q^{2} \rightarrow Q^{2}$ exchange intensities (Fig. 8b) large deviations between experiment and simulations of both the alternating and clustering structural models were observed, with only the random distribution model predicting the correct dependence as a function of modifier content. As $\Delta E$ decreased for the alternating and clustering models the resulting structures become more random, with corresponding improvement in the fit to the experimental data. These results demonstrate that pure alternating or clustering structures are not present in lithium ultraphosphate glasses.

\subsection{Double quantum $2 D$ exchange experiments}

While there have been several double-quantum (2Q) ${ }^{31} \mathrm{P}$ MAS NMR $2 \mathrm{D}$ exchange experiments reported for phosphate systems, the non-quantitative $2 \mathrm{Q}$ experiment has hindered analysis of connectivity distributions in glasses. Recently Witter and co-workers[27] presented a novel approach utilizing the chemical shifts obtained from $2 Q^{31} \mathrm{P}$ NMR experiments to deconvolute the simple 1D MAS ${ }^{31} \mathrm{P}$ spectra, for a series of calcium phosphate glasses. Using the quantitative intensities of 1D MAS experiments, an estimate of the phosphate tetrahedron connectivity distributions for these calcium phosphate glasses was obtained. More detailed information is available from the $2 \mathrm{Q}$ experiment in comparison to the RFDR exchange experiments, since individual $f\left(\mathrm{Q}^{n, i j k}\right)$ values can be directly measured. The reported experimental 
distributions for the calcium phosphate glasses are shown in Fig. 9, along with the predictions for a random, alternate $\left(\Delta E=0.25 k_{B} T\right)$ and a cluster model $\left(\Delta E=0.25 k_{B} T\right)$. The experimental observation of $f\left(\mathrm{Q}^{2,12}\right)$ species for these glasses in comparison to the $f\left(\mathrm{Q}^{2,12}\right)$ for a pure alternating structure shown in Fig. 5a, and the $f\left(Q^{2,12}\right)$ for a pure clustering structure in Fig. 7a, immediately eliminates both a pure alternating structure and a pure clustering structure, and demonstrates that the bonding distribution is predominantly random. Observations of $2 Q N M R Q^{2}-Q^{1}$ connectivities, along with $Q^{2}-Q^{2}$ and $Q^{1}-Q^{1}$ connectivities in sodium phosphate glasses, [26] and silver iodine-phosphate glasses[22] has also been reported. Those $2 \mathrm{Q}$ NMR results also prove that for these glasses, pure alternating or cluster type structures are not present, but instead must contain some amount of random connectivity. Preliminary 2Q NMR investigations of zinc phosphate glasses suggest that an alternating $Q^{n}$ structure may be the dominant structural motif, but a more detailed analysis of the NMR results is warranted.[34]

In attempt to improve the fit with the observed experimental distribution in the calcium phosphate glasses reported by Witter et al.,[27] simulations for smaller $\Delta E$, representing mixed random-alternate or random-cluster structural types, are shown in Fig. 9. The $f\left(\mathrm{Q}^{2, i j}\right)$ distributions have the largest deviation from the random connectivity predicted fractions (Fig. 9a), but neither the alternating or cluster model can improve these deviations for all three $f\left(\mathrm{Q}^{2, i j}\right)$ fractions. The low energy cluster model $\left(\Delta E=0.25 k_{B} T\right)$ improves the fit of $f\left(\mathrm{Q}^{2.22}\right)$ and $f\left(\mathrm{Q}^{2.12}\right)$ fraction, but offers no improvement in the fit of $f\left(Q^{2,11}\right)$. Similarly the low-energy alternate model $(\Delta E=0.25$ $\left.k_{B} T\right)$ improves the fit of $f\left(\mathrm{Q}^{2,12}\right)$ fraction, but under-estimates the $f\left(\mathrm{Q}^{2,22}\right)$ fraction and overestimates the $f\left(\mathrm{Q}^{2,11}\right)$ fraction at larger modifier concentration (Fig. 9a). For the $f\left(\mathrm{Q}^{1, i}\right)$ distributions (Fig. 9b) predictions by the random model provides the best fit. These results also demonstrate that the variations in the model distributions ( in this case $f\left(\mathrm{Q}^{2, i i}\right)$ and $f\left(\mathrm{Q}^{1, i}\right)$ ) as a function of modifier concentration are dependent on the details of the model being investigated. 
For these calcium phosphate glasses it is also possible to envision a structure where the $\mathrm{Q}^{2}$ connectivity is described by a mixed cluster-random model, while the $\mathrm{Q}^{1}$ connectivity is described by a pure random distribution model. Such structural models involving changes in the connectivity pattern as a function of $\mathrm{Q}^{n}$ can quickly become very complex. With the limited amount of experimental data directly addressing the next-nearest neighbor connectivity in phosphate glasses, such $\mathrm{Q}^{n}$ dependent models were not pursued here. Given the estimated 25\% error reported for the 2 Q NMR experimental distributions,[27] the connectivity distribution results for the calcium phosphate glass series are most accurately described by a random model.

\section{Conclusions}

Comparison of the distribution of next-nearest neighbor $\mathrm{Q}^{\mathrm{n}}$ connectivities from Monte Carlo simulations and experimental 2D RFDR and double-quantum ${ }^{31} \mathrm{P}$ NMR experiments has allowed a critical evaluation of different structural models including random, alternating and clustering bonding distributions. The variation in polyhedron connectivity as a function of the relative difference in bond energies for the structural models investigated enable the uniqueness of the various models to be determined. Comparison of the MC simulations and the experimental distributions demonstrate that for both the calcium pyrophosphate glass series and the lithium ultraphosphate glass series, the phosphate polyhedron connectivity is most accurately described by a random distribution.

\section{Acknowledgements}

Sandia is a multiprogram laboratory operated by Sandia Corporation, a Lockheed Martin Company, for the United States Department of Energy under Contract DE-AC04-94AL85000.

The author is also indebted to the helpful comments and suggestions of Dick Brow. 


\section{References}

[1] D.E. Day, Z. Wu, C.S. Ray, P. Hrma, J. Non-Cryst. Solids 241 (1998) 1.

[2] R.K. Brow, T.M. Alam, D.R. Tallant, R.J. Kirkpatrick, MRS Bulletin 23 (1998) 63.

[3] M.J. Weber, J. Non-Cryst. Solids 123 (1990) 208.

[4] J.A. Wilder, J. Non-Cryst. Solids $38 / 39$ (1980) 879.

[5] A. Mogusmilankovic, M. Rajic, A. Drasner, R. Trojko, D.E. Day, Phys. Chem. of Glasses 39 (1998) 70 .

[6] H. Eckert, Progress in NMR Spectroscopy 24 (1992) 159.

[7] J.W. Zwanziger, Int. Review Phys. Chem. 17 (1998) 65.

[8] R.J. Kirkpatrick, R.K. Brow, Solid State Nuclear Magnetic Resonance 5 (1995) 9.

[9] R.K. Brow, R.J. Kirpatrick, G.L. Turner, J. Non-Cryst. Solids 116 (1990) 39.

[10] R.K. Brow, C.C. Phifer, G.L. Turner, R.J. Kirkpatrick, J. Am. Ceram. Soc. 74 (1991) 1287.

[11] R.K. Brow, D.R. Tallant, J.J. Hudgens, S.W. Martin, A.D. Irwin, J. of Non-Crystalline Solids 177 (1994) 221.

[12] R.K. Brow, R.J. Kirkpatrick, G.L. Turner, J. Amer. Ceramic Society 76 (1993) 919.

[13] T.M. Alam, S. Conzone, R.K. Brow, T.J. Boyle, J. Non-Cryst. Solids 258 (1999) 140.

[14] R.K. Brow, L. Kovacic, R.E. Loehman, Ceram. Trans. 70 (1996) 177.

[15] T.M. Alam, J. McLauglin, C.C. Click, S. Conzone, R.K. Brow, T.J. Boyle, J.W. Zwanziger, J. Phys. Chem. (2000) (In Press).

[16] C. Jäger, M. Feike, R. Born, H.W. Spiess, J. Non-Cryst. Solids 180 (1994) 91.

[17] R. Born, M. Feike, C. Jäger, H.W. Spiess, Z. Naturforsch 50a (1995) 169.

[18] P. Hartman, J. Vogel, C. Jäger, Ber. Bunsenges. Phys. Chem. 100 (1996) 1658.

[19] P. Hartmann, C. Jana, J. Vogel, C. Jäger, Chem. Phys. Lett. 258 (1996) 107.

[20] C. Jäger, P. Hartmann, G. Kunath-Fandrei, O. Hirsch, P. Rehak, J. Vogel, M. Feike, H.W.

Spiess, K. Herzog, B. Thomas, Ber. Bunsenges. Phys. Chem. 100 (1996) 1560.

[21] T.M. Alam, R.K. Brow, J. Non-Crystalline Solids 223 (1998) 1. 
[22] K.K. Olsen, J.W. Zwanziger, P. Hartmann, C. Jäger, J. Non-Cryst. Solids 222 (1997) 199.

[23] M. Feike, D.E. Demco, R. Graf, J. Gottwald, S. Hafner, H.W. Spiess, J. Magn. Reson., Ser. A $122(1996) 214$.

[24] M. Feike, R. Graf, I. Schnell, C. Jäger, H.W. Spiess, J. Am. Chem. Soc. 118 (1996) 9631.

[25] W.A. Dollase, M. Feike, H. Förster, T. Schaller, I. Schnell, A. Sebald, S. Steuernagel, J. Am. Chem. Soc. 119 (1997) 3807.

[26] M. Feike, C. Jäger, H.W. Spiess, J. Non-Cryst. Solids 223 (1998) 200.

[27] R. Witter, P. Hartmann, J. Vogel, C. Jäger, Solid State Nuclear Magnetic Resonance 13 (1998) 189.

[28] D. Sprenger, H. Bach, W. Meisel, P. Gütlich, J. Non-Cryst. Solids 159 (1993) 187.

[29] J. van Wazer, Phosphorus and its Compounds; Interscience: New York, 1958.

[30] R.K. Brow, D.R. Tallant, S.T. Myers, C.C. Phifer, J. Non-Cryst. Solids 191 (1995) 45.

[31] This nomenclature differs slightly from that presented in Ref. [21], but was chosen to be consistent with the nomenclature used to describe the connectivities obtained from double quantum NMR (See Ref. [27]).

[32] Eqn. (A.8) in Ref. [21] is in error and should read $b_{\mathrm{Q}^{1}}=\frac{p_{\mathrm{Q}^{\mathrm{i}}}}{2-p_{\mathrm{Q}^{1}}}$. This correct relationship was used in deriving $b_{\mathrm{Q}^{1}}$, Eqn. $9 \mathrm{~b}$.

[33] K. Meyer, J. Non-Cryst. Solids 209 (1997) 227.

[34] B. Tischendorf, J.W. Wiench, M. Pruski, J.U. Otaigbe, 15 th University Conference on Glass Science, Rolla, MO, 1999. 


\section{Figures}

Fig. 1 The phosphate tetrahedron distributions, a) $f\left(\mathrm{Q}^{3, j i k}\right)$ and b) $f\left(\mathrm{Q}^{2, j j}\right)$ for the binary phosphate glass, $x \mathrm{R}_{2 / \mathrm{n}}^{\mathrm{n}+} \mathrm{O} \cdot(1-x) \mathrm{P}_{2} \mathrm{O}_{5}, 0 \leq x<0.5$. Details are given in the text.

Fig. 2 The phosphate tetrahedron distributions, a) $f\left(\mathrm{Q}^{2, i j}\right)$ and b) $f\left(\mathrm{Q}^{1 . j}\right)$ for the binary phosphate glass, $x \mathrm{R}_{2 / \mathrm{n}}^{\mathrm{n}+} \mathrm{O} \cdot(1-x) \mathrm{P}_{2} \mathrm{O}_{5}, 0.5 \leq x<0.67$.

Fig. 3 The phosphate tetrahedron distributions $f\left(\mathrm{Q}^{3, j j k}\right)$ for a alternating $\mathrm{Q}^{n}-\mathrm{Q}^{n^{\prime}}$ structural model in the binary phosphate glass, $x \mathrm{R}_{2 / \mathrm{n}}^{\mathrm{n}+} \mathrm{O} \cdot(1-x) \mathrm{P}_{2} \mathrm{O}_{5}, 0 \leq x<0.5$, for a) $\Delta E=10 k_{B} T$, b) $\Delta E=5$ $k_{B} T$, c) $\Delta E=1 k_{B} T$ and d) $\Delta E=0.5 k_{B} T$. Details are given the text.

Fig. 4 The phosphate tetrahedron distributions $f\left(\mathrm{Q}^{2 . j j}\right)$ for an alternating $\mathrm{Q}^{n}-\mathrm{Q}^{n^{\prime}}$ structural model in the binary phosphate glass, $x \mathrm{R}_{2 / \mathrm{n}}^{\mathrm{n}+} \mathrm{O} \cdot(1-x) \mathrm{P}_{2} \mathrm{O}_{5}, 0 \leq x<0.5$, for a) $\Delta E=10 k_{B} T$, b) $\Delta E=5 k_{B} T$, c) $\Delta E=1 k_{B} T$ and d) $\Delta E=0.5 k_{B} T$.

Fig. 5 The phosphate tetrahedron distributions for an alternating $\mathrm{Q}^{n}-\mathrm{Q}^{n^{\prime}}$ structural model in the binary phosphate glass, $x \mathrm{R}_{2 / \mathrm{n}}^{\mathrm{n}+} \mathrm{O} \cdot(1-x) \mathrm{P}_{2} \mathrm{O}_{5}, 0.5 \leq x<0.67$, for a) $f\left(\mathrm{Q}^{2, i j}\right)$ with $\Delta E=1 k_{B} T$, b) $\left.f\left(\mathrm{Q}^{2, i j}\right) \Delta E=0.5 k_{B} T, \mathrm{c}\right) f\left(\mathrm{Q}^{1, i}\right)$ with $\Delta E=1 k_{B} T$ and d) $f\left(\mathrm{Q}^{1, i}\right)$ with $\Delta E=0.5 k_{B} T$. Details are given the text.

Fig. 6 The phosphate tetrahedron distributions for a structural model incorporating $Q^{n}$ clustering for the binary phosphate glass, $x \mathrm{R}_{2, \mathrm{n}}^{\mathrm{n}+} \mathrm{O} \cdot(1-x) \mathrm{P}_{2} \mathrm{O}_{5}, 0 \leq x<0.5$; a) $f\left(\mathrm{Q}^{3, i j k}\right)$ with $\left.\Delta E=1 k_{B} T, \mathrm{~b}\right)$ 
$f\left(\mathrm{Q}^{3, i j k}\right)$ with $\left.\Delta E=0.5 k_{B} T, \mathrm{c}\right) f\left(\mathrm{Q}^{3, i j k}\right)$ with $\left.\Delta E=0.25 k_{B} T, \mathrm{~d}\right) f\left(\mathrm{Q}^{2, j j}\right)$ with $\Delta E=1.0 k_{B} T$, e) $f\left(\mathrm{Q}^{2, i j}\right)$ with $\Delta E=0.5 k_{B} T$ and e) $f\left(Q^{2, i j}\right)$ with $\Delta E=0.25 k_{B} T$. Details are given the text.

Fig. 7 The phosphate tetrahedron distributions for a structural model incorporating $\mathrm{Q}^{n}$ clustering in the binary phosphate glass, $x \mathrm{R}_{2 / \mathrm{n}}^{\mathrm{n}+} \mathrm{O} \cdot(1-x) \mathrm{P}_{2} \mathrm{O}_{5}, 0.5 \leq x<0.67$; a) $f\left(\mathrm{Q}^{2, i j}\right)$ with $\left.\Delta E=1 k_{B} T, \mathrm{~b}\right)$ $f\left(\mathrm{Q}^{2, i j}\right)$ with $\left.\Delta E=0.5 k_{B} T, \mathrm{c}\right) f\left(\mathrm{Q}^{2, i j}\right)$ with $\Delta E=0.25 k_{B} T$, d) $f\left(\mathrm{Q}^{1, i}\right)$ with $\Delta E=1.0 k_{B} T$, e) $f\left(\mathrm{Q}^{1, i}\right)$ with $\Delta E=0.5 k_{B} T$ and e) $f\left(\mathrm{Q}^{1, i}\right)$ with $\Delta E=0.25 k_{B} T$.

Fig. 8 A comparison between the experimental $(\bullet, O)$ RFDR 2D NMR exchange cross-peak intensities and the theoretical predictions for a random distribution model, an $\mathrm{Q}^{n}-\mathrm{Q}^{\prime \prime}$ alternating structural model $\left(\Delta E=10 k_{B} T\right)$, and an $\mathrm{Q}^{\prime \prime}$ clustering model $\left(\Delta E=5 k_{B} T\right)$. In a) the $\mathrm{Q}^{3} \rightarrow \mathrm{Q}^{3}$ intensities ( the $Q^{2} \rightarrow Q^{3}$ intensities $\left(O\right.$, solid lines) and the $Q^{2} \rightarrow Q^{2}$ intensities $(O$, dotted lines) are presented.

Fig. 9 A comparison of the $f\left(\mathrm{Q}^{2, i j}\right)$ and $f\left(\mathrm{Q}^{1, i}\right)$ connectivity distributions determined for calcium orthophosphate glasses by $2 \mathrm{Q} 2 \mathrm{D}{ }^{31} \mathrm{P}$ MAS NMR and the predicted distributions for a random $\left(\longrightarrow\right.$, alternating, $\Delta E=0.25 k_{B} T(\bullet \bullet \bullet \bullet)$, and a cluster model, $\Delta E=0.25 k_{B} T(\longrightarrow \bullet)$. In a) the experimental $f\left(\mathrm{Q}^{2,22}\right)(\odot), f\left(\mathrm{Q}^{2,12}\right)(\mathrm{O})$ and $f\left(\mathrm{Q}^{2,11}\right)(\boldsymbol{\square})$ values are shown, while in b) $f\left(\mathrm{Q}^{1,2}\right)$ $(\odot), f\left(Q^{1,1}\right)(O)$ are presented. The concentrations were calculated from relative intensities reported in Ref. [27]. 


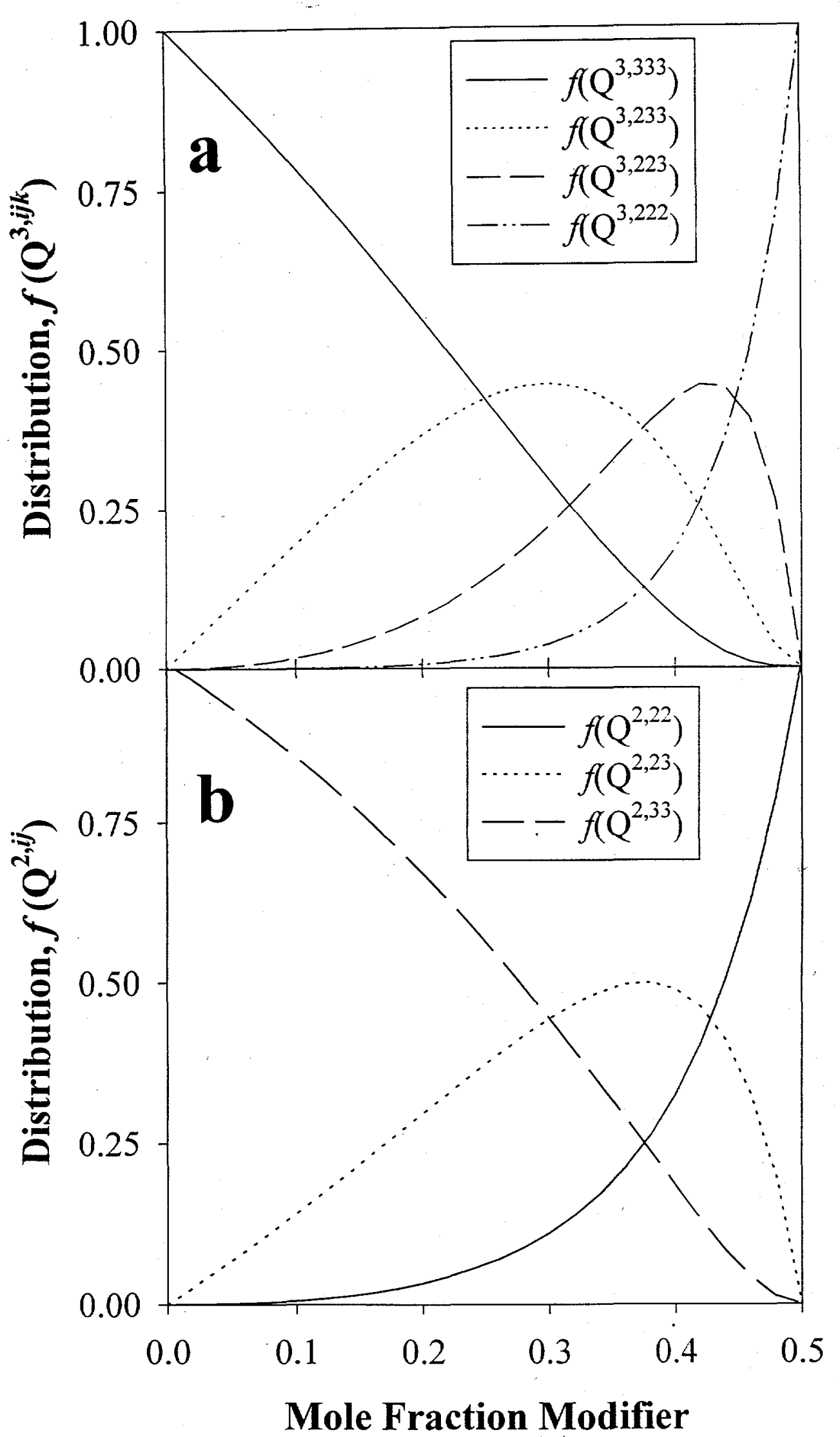

Alam Fig. 1 


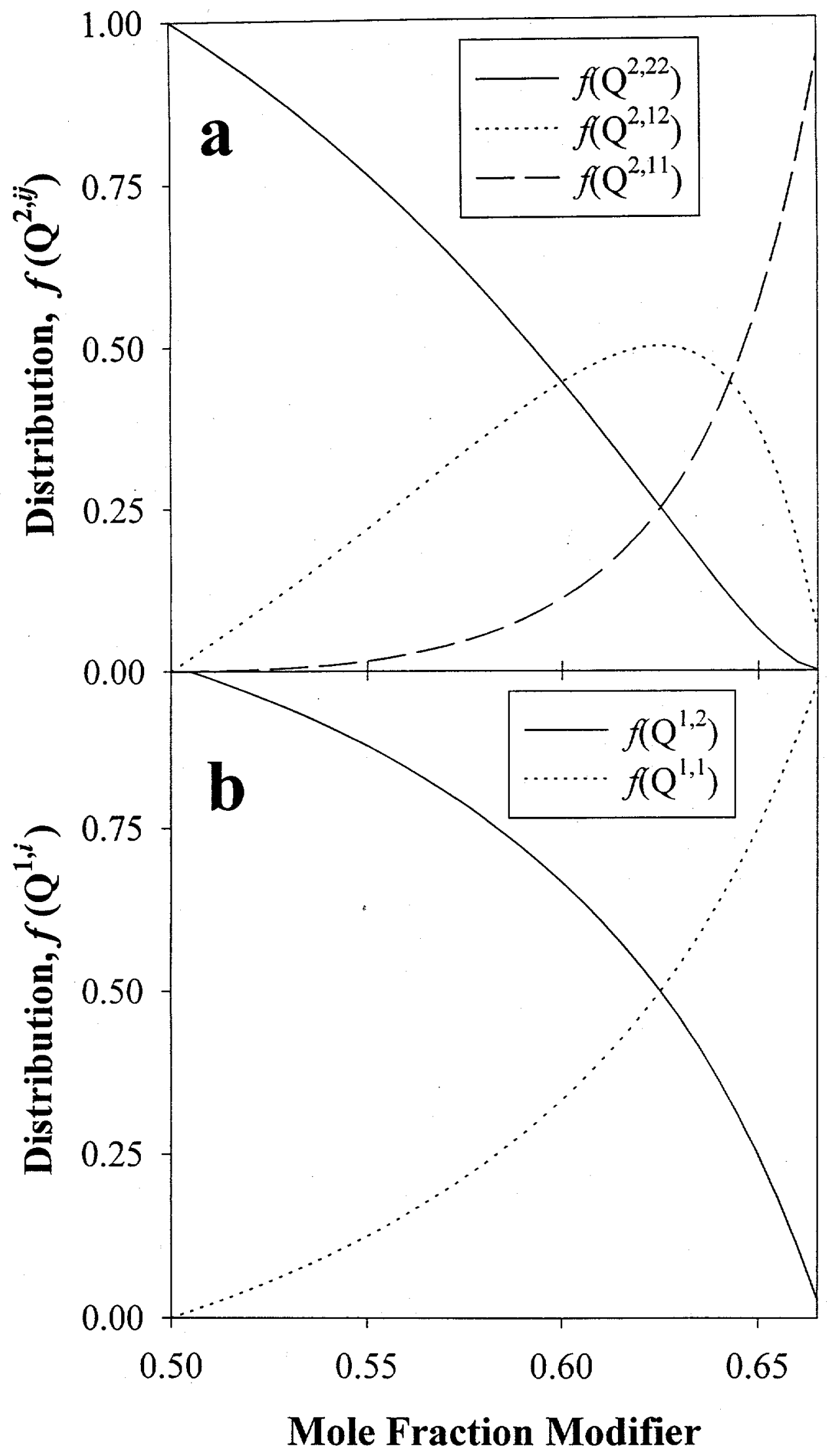

Alam Fig. 2 


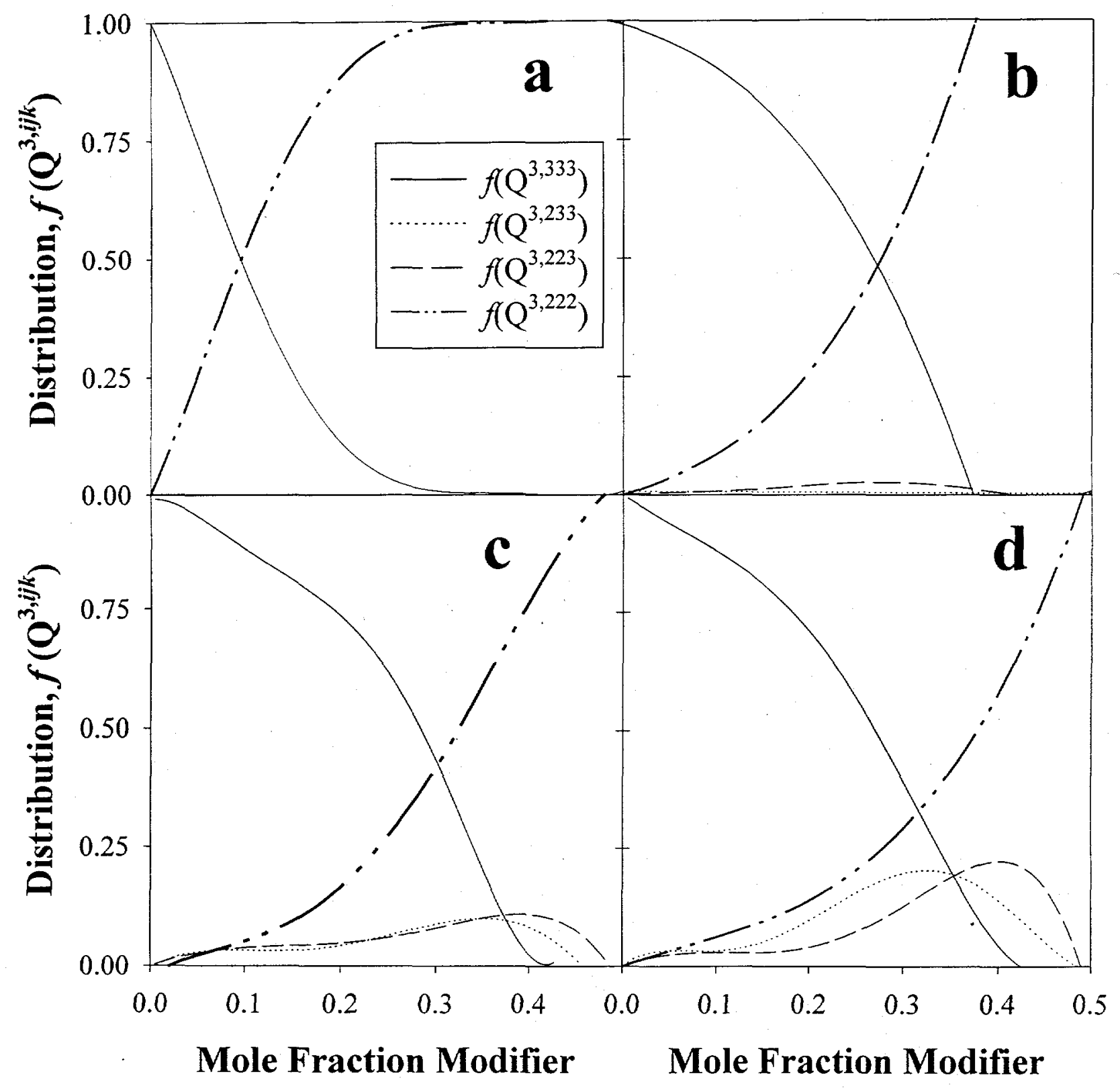

Alam Fig. 3 


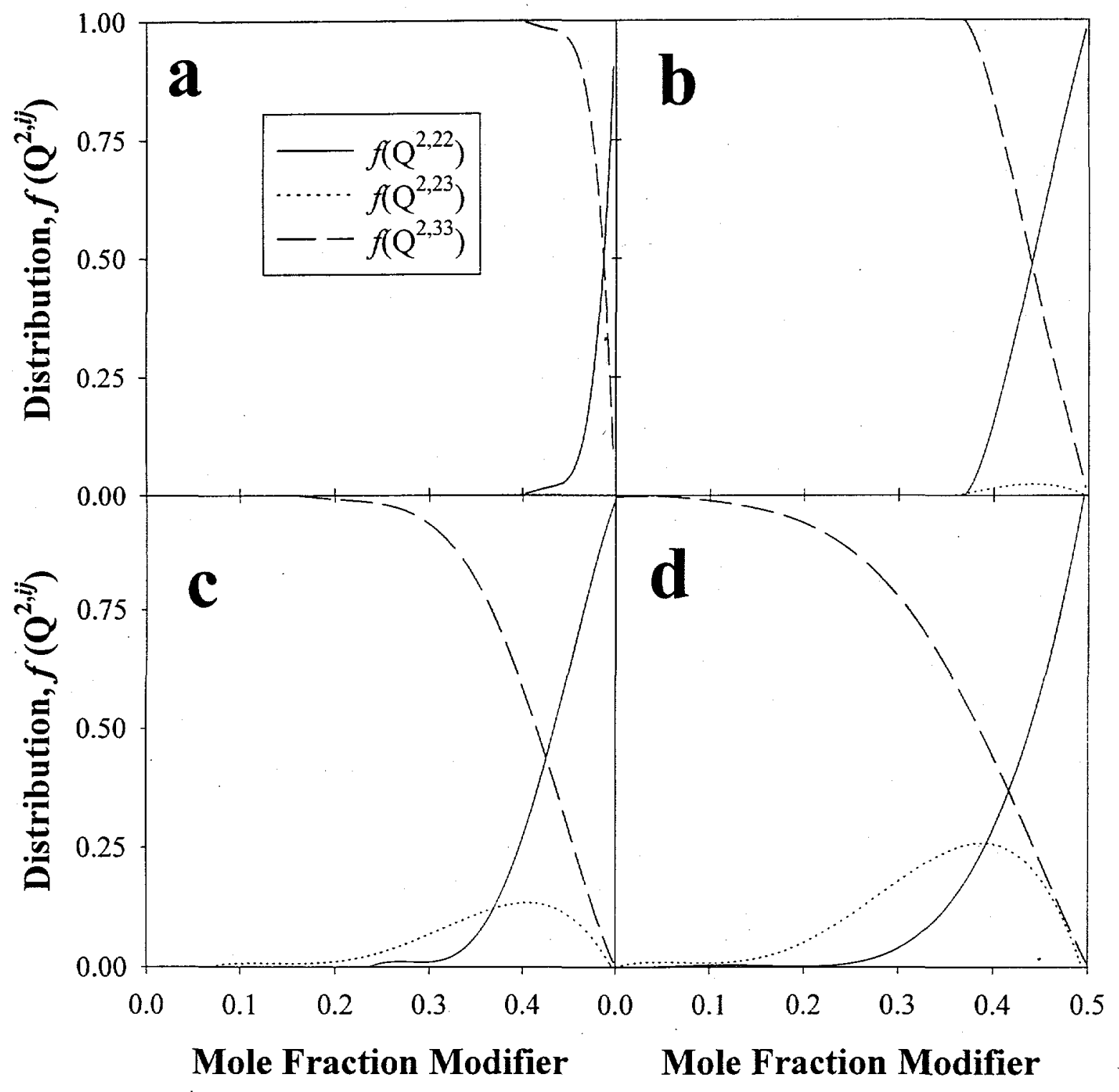

AlamFig. 4 


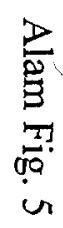

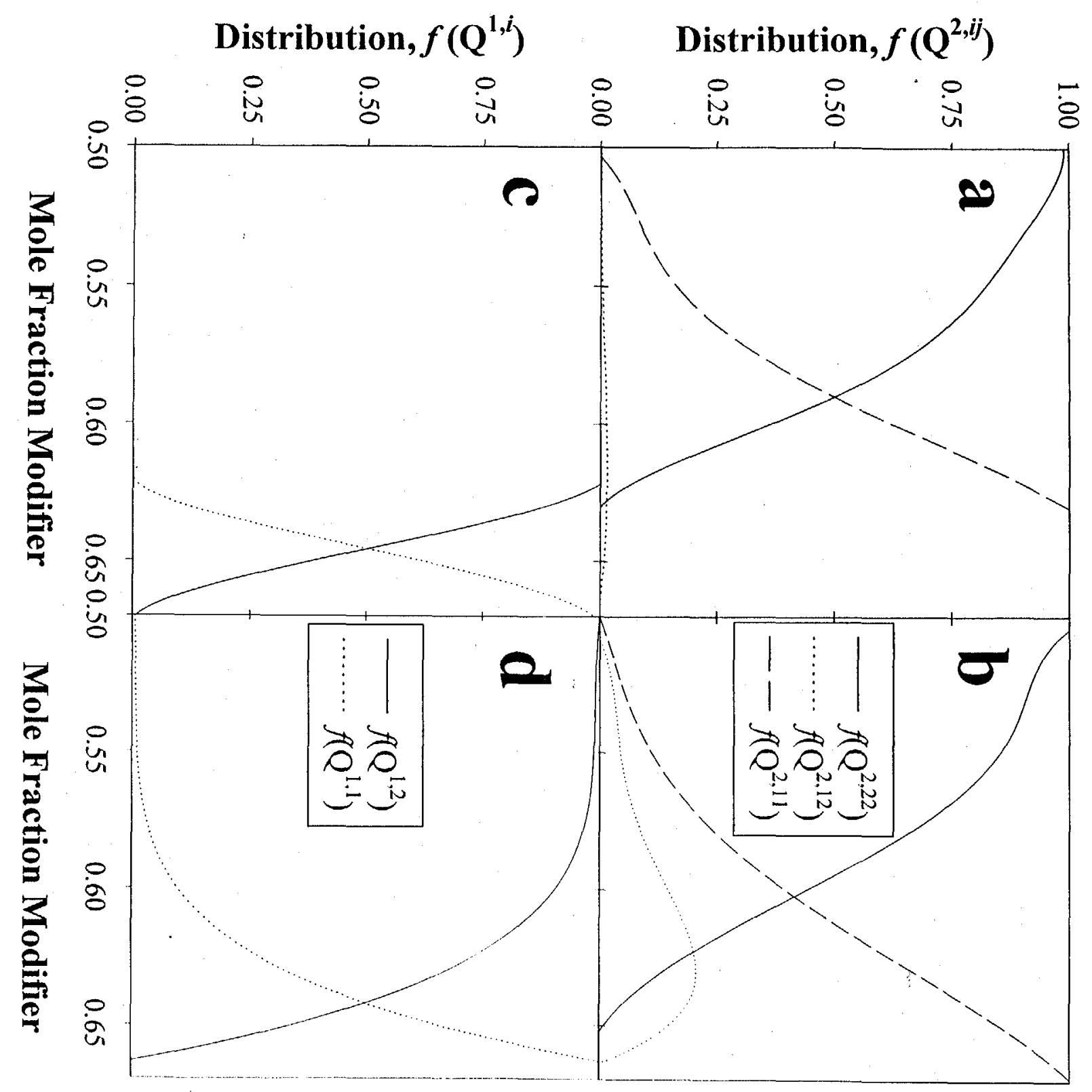



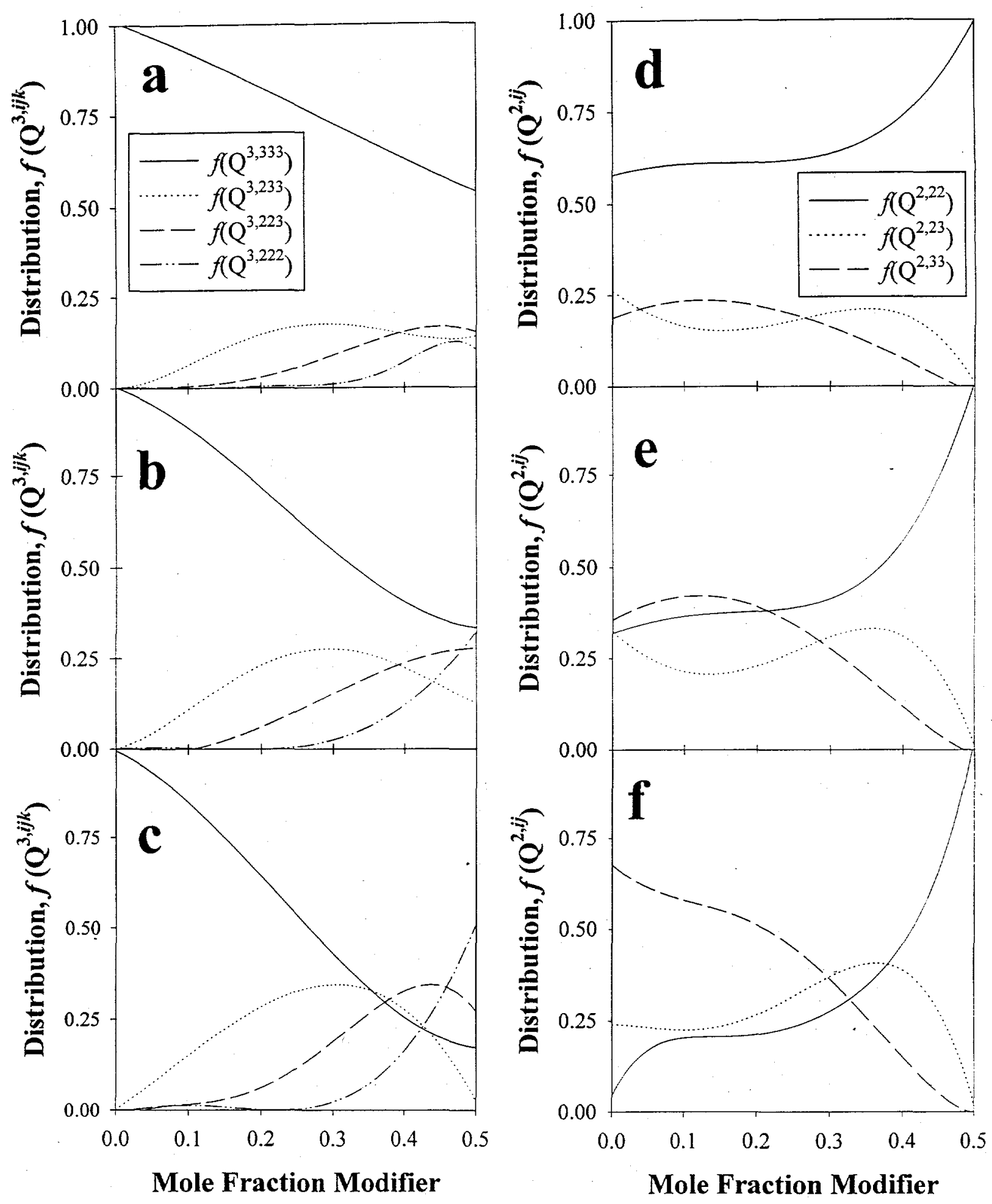

Alam Fig. 6 

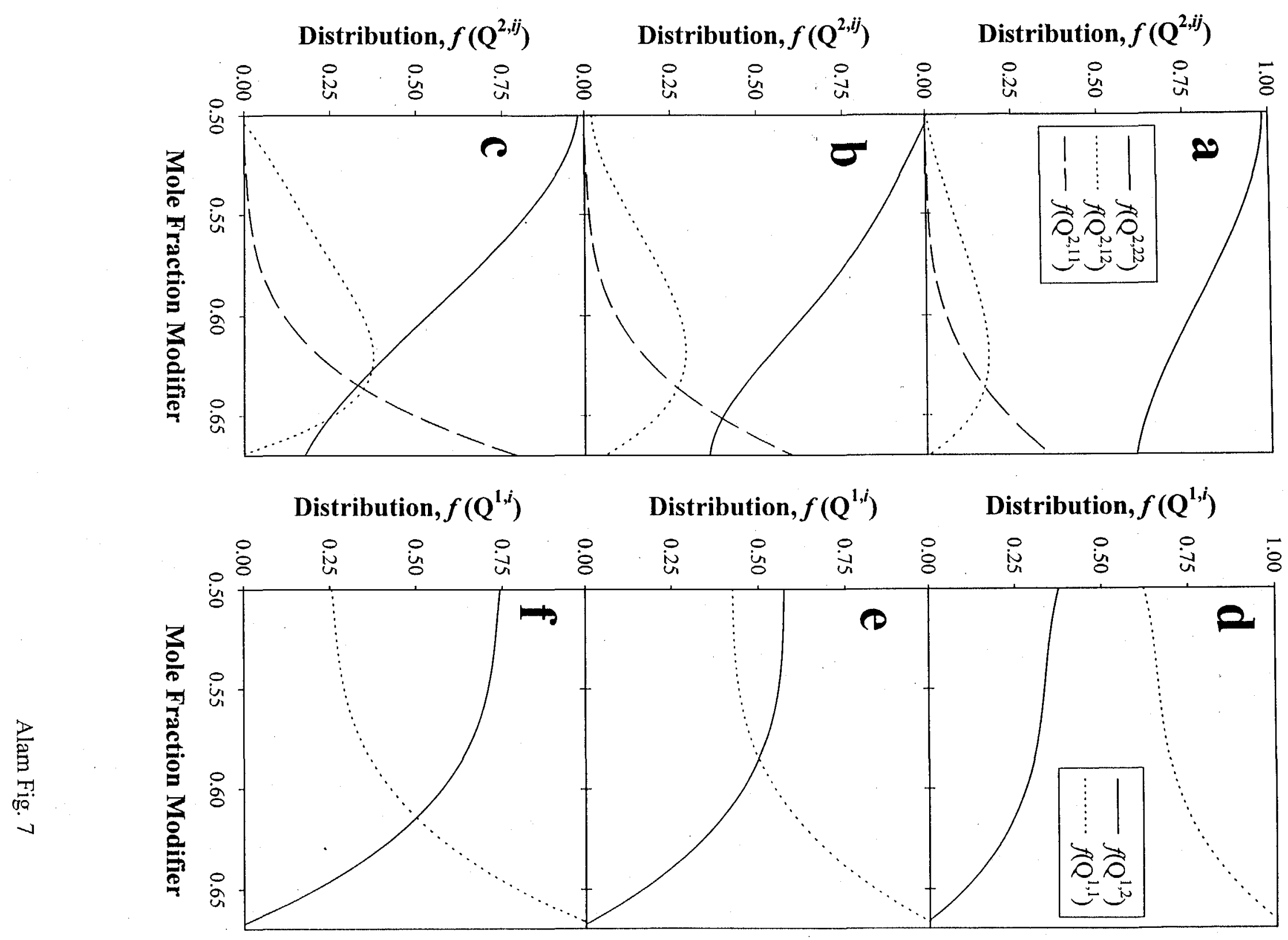


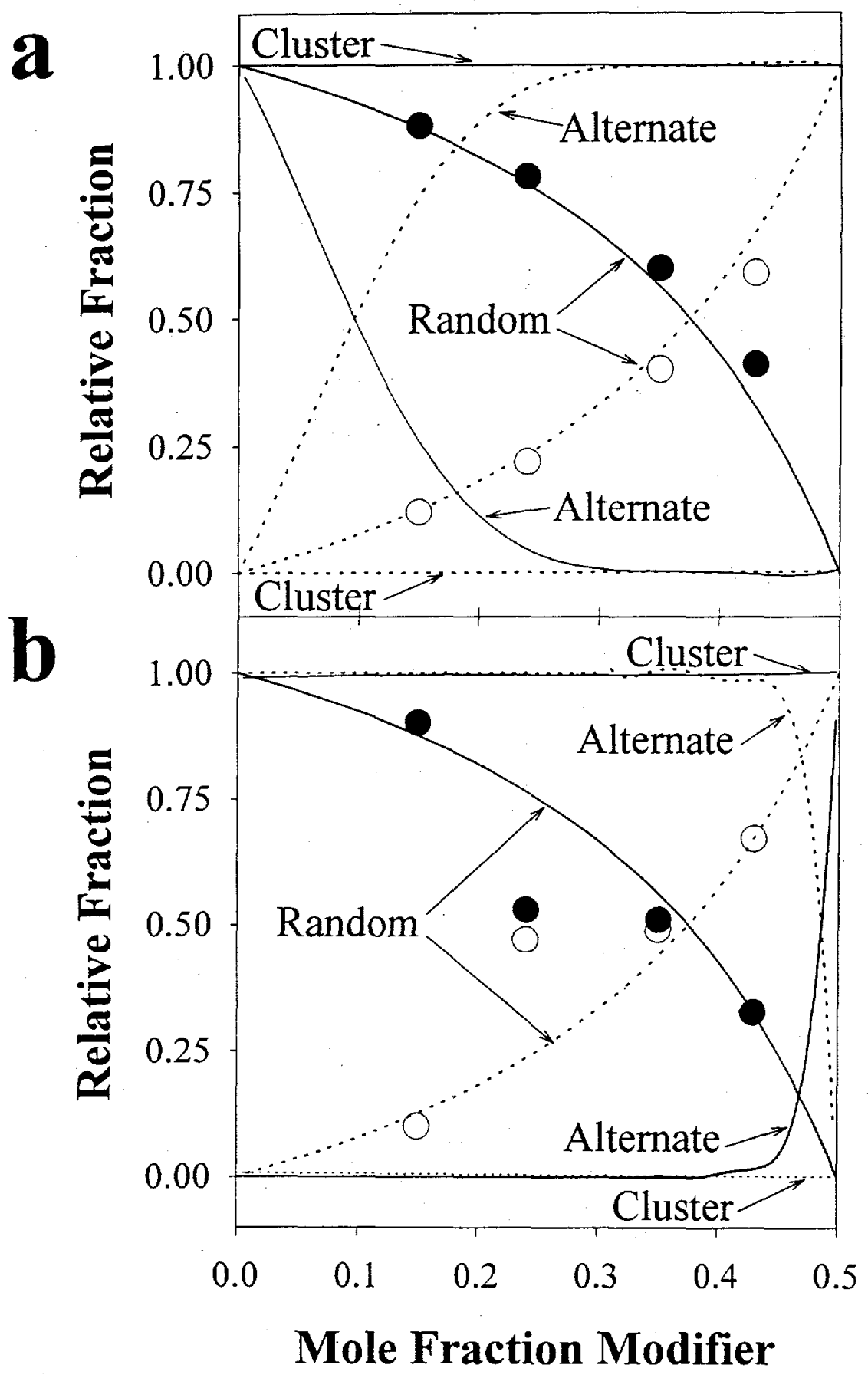

Alam Fig. 8 


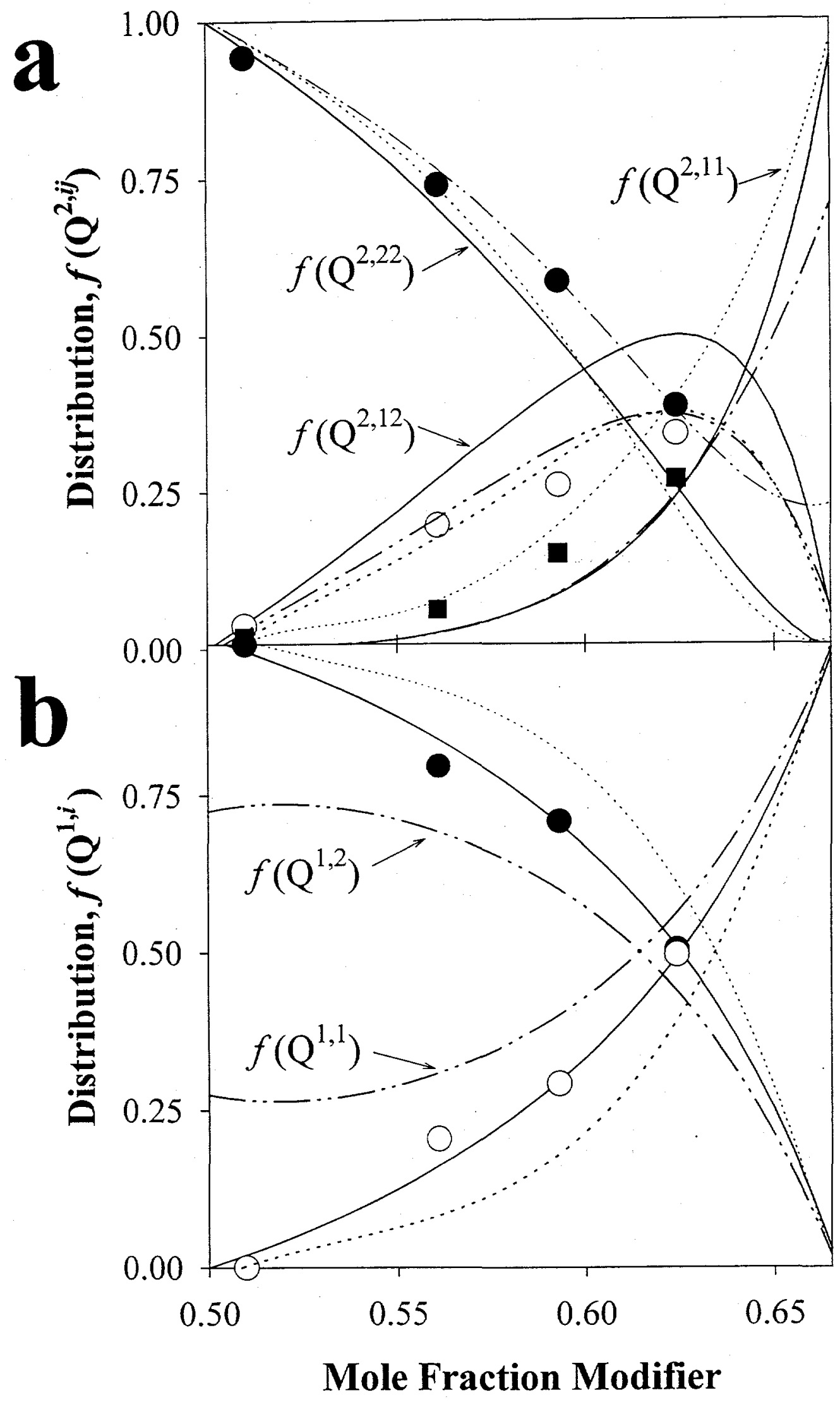

Alam Fig. 9 